\title{
WWW Recycling for a Better World
}

Stefano Ferretti ${ }^{1}$, Marco Furini ${ }^{2}$, Claudio E. Palazzi ${ }^{* 3}$, Marco Roccetti $^{1}$, Paola Salomoni ${ }^{1}$

Web 2.0 is affecting the structure of our society by creating new spaces of freedom, giving voice to any opinion, easing interpersonal relationships, and encouraging the creation of collaborating collectivities. Technologies such as blog, podcasting, wiki, and news feeds have the power to transform every user from a mere information consumer to a potential producer, from a spectator to an actor. The success of YouTube, MySpace, Facebook, and Wikipedia is under everybody's eyes. Generally speaking, Web 2.0 is seen as a powerful tool that, by exploiting Internet technologies, supplies services to the society, helping the entire socio-cultural system to develop and to move toward a democratic direction.

Unfortunately, this imagery is partially distorted: first, Web 2.0 and Internet technologies are differently accessible by different parts of the society and, second, as many sociologists pointed out, their applications often appeal to users' egoistic purposes and self-celebration spirit [1]. Although we cannot dismiss the value of current Web 2.0 applications, we are convinced that there is a much more valuable potential that has not been exploited yet. By comparing the immense benefits that Web 2.0 could bring to the whole society, with its factual employment, one could provocatively change the meaning of the acronym WWW into World Wide Waste. We are hence convinced that it is necessary to redesign the utilization paradigm of Web 2.0 and, in general, of the Internet in order to recycle

\footnotetext{
*Corresponding author. E-Mail: cpalazzi@math.unipd.it

${ }^{1}$ Dipartimento Scienze dell'Informazione - Università di Bologna, Mura Anteo Zamboni 7, 40127 Bologna, Italy.

Email: \{sferrett, roccetti, salomoni\}@cs.unibo.it

${ }^{2}$ Dipartimento di Informatica - Università del Piemonte-Orientale, Via Bellini 25/G, 15100 Alessandria, Italy. Email: furini@mfn.unipmn.it

${ }^{3}$ Dipartimento di Matematica Pura ed Applicata - Università di Padova, Via Trieste 63, 35121 Padova, Italy. Email: cpalazzi@math.unipd.it
} 
unused parts of Web 2.0 into altruistic bricks that could be appropriately rerouted and composed for alternative, unselfish employment.

\section{AN UNEXPLOITED POTENTIAL}

In this article, we provide guidelines for a new interpretation of potentialities cocooned by Web 2.0 technologies: by finding and recycling unutilized resources over the Internet, it is possible to create an integrated platform that reroutes them toward an altruistic use. To create a more powerful platform, both hardware and software entities are considered as "resources" (e.g., data, programs, processor's cycles, storage capacity, cameras, communication capabilities). This integrated platform, along with a simple graphical interface that hides all the technological difficulties, would help creating and developing novel services and applications that were not originally provided by either sources. Therefore, the platform would increase the social solidarity and favor the establishment of "a better world", as provocatively announced in the title.

Before presenting the new interpretation of Web 2.0 potentialities, let us analyze why the current vision of Web 2.0, as a powerful tool for the development of the entire society, may be questioned.

First of all, Internet technologies at the core of Web 2.0 are differently accessible by different parts of the society. This might create social discrimination between who has access to these technologies and who has not $[2,3]$. This phenomenon (known as digital divide) is mainly due to: linguistic marginalization (e.g., illiteracy impedes the approach to information); structure marginalization (e.g., unavailable resources can deny the use of technology); technological marginalization (e.g., continuous technological modernization can be problematic for economic-cultural reasons); and social marginalization (e.g., peo-

ple affected by physical or health problems are excluded from the use of many technologies). Although not new, this phenomenon is nowadays particularly critical, as many sociologists agree that the current Information Society is in the phase where the development of particular applications (e.g., Web 2.0) can generate a mutation of the society itself [4].

Secondly but not in order of importance, the success of Web 2.0 mainly attracts applications motivated by ego-centered behaviors (e.g., enrichment and self-celebration). It is worth noting that there is a long sociological and philosophical debate concerning whether a simple action can be seen as altruism or egoism (is it egoism or altruism to have a dog? is it egoism or altruism to write a book review on a website?) [5]. Far from settling this debate, we simply observe that the vast potential of Web 2.0 technologies is far from being largely exploited. 
At the base of this technology's waste there is the fact that we are still mislead by the belief that means are neutral in principle and that it is their use that makes them positive or negative. This is a naive interpretation; instead, our ambitious goal is to generate an integrated platform that could be resilient to a pure egoistic use. This platform will capture unutilized resources and reroute them toward an altruistic use.

We can consider this platform as a manager of resources that can be located all over the world; its aim is that of retrieving and combining them when needed so as to provide altruistic services.

The idea is that even a selfish use of the technology, in order to achieve personal objectives or to have fun, can be rerouted by a layer devoted to the management and diffusion of collective resources so as to make emerging an altruistic purpose. To factually deploy such a platform, five main steps are required:

1. Integrate the most widely used communication platforms, Internet, mobile, and television, to generate a global broadband and broadcast communication scenario, able to guarantee to every member of the society an easy access to information independently from the used device.

2. Deploy a system able to collect and organize (categorize) resources made available by the various users, and to have them utilizable in case of (altruistic) need.

3. Motivate potential users, even through selfish advantages, so that they will subscribe the system and render available part of their resources in case of need for an altruistic aim.

4. Provide a programmable environment so that the development of altruistic applications would be as easy as using a single integrated tool.

5. Create new altruistic services and applications, by which categorized resources can be used for social purposes thanks also to their diffusion in various contexts.

\section{PRACTICAL CASE STUDIES}

As representative examples, two case studies follow (also depicted in Figure 1), showing how a careful and integrated use of new technologies permits the development of new altruistic applications.

Example 1. Remote monitoring of sick people is an application more and more diffused. Think of a remotely monitored patient who experiences a sudden and unexpected worsening of his health conditions while walking in a park. The arrival of doctors called by the remote monitoring station could 


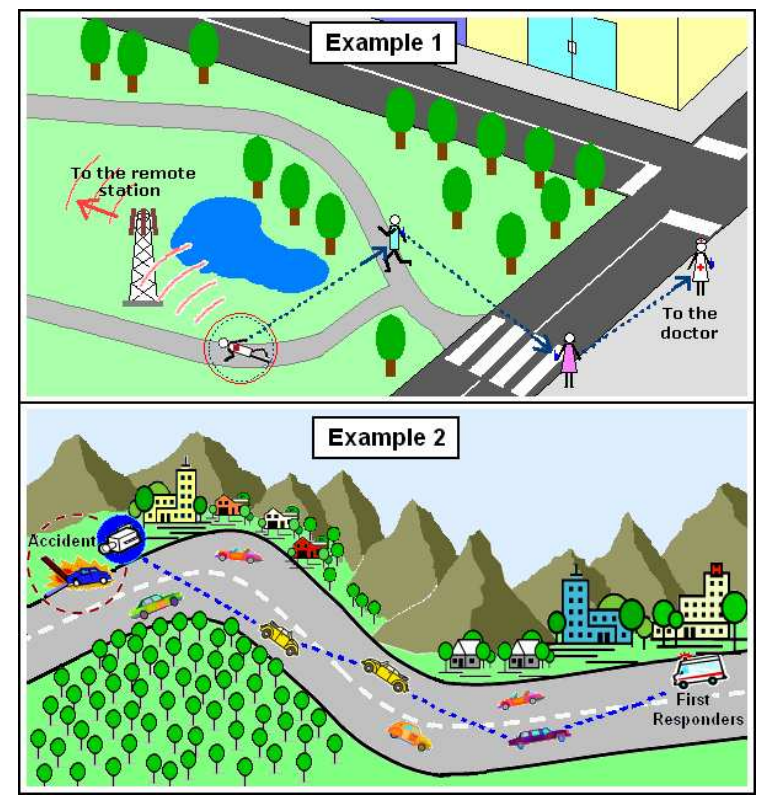

Figure 1: Exemplars of altruistic applications deployable thanks to the proposed integrated platform.

be not timely enough. Instead, it would be more effective to also exploit the various devices owned by people in the surroundings to propagate the aid-request until reaching the smart phone of a doctor in the neighborhood, who could promptly take action while waiting for the ambulance. Therefore, paradoxically, a runner who goes jogging for personal interest becomes an altruistic subject by putting on hand his fancy technological resources (e.g., the communication capabilities of his iPhone).

Example 2. Consider a crisis scene in a city, e.g., a street accident or a terrorist attack. In this scenario, it would be useful to provide first responders with real-time pictures/videos of the emergency while still driving toward the crisis area. Devices utilized for this purpose could be security cameras in the area or any other camera-endowed device (e.g., a cellphone) possessed by people in proximity of the emergency area. Both commands to activate the device and generated pictures/videos can be sent through the vehicular network directly to the vehicle of first responders, or reach them through the Internet and the cellular network. In any case, elements that are typically considered of disturbance in emergency situations (e.g., vehicles that increase the traffic and slow down the arrival of ambulances, "curious" people who typically interfere with aid operations) can turn out to be of help. 
To practically explain how to include a selfish application into our altruistic vision, we consider two selfish applications showing how they can be part of a wider, altruistic aim.

Selfish application 1. Ad-Torrent is a digital billboard system that provides users with location based advertisement messages $(A d s)$, guided by a particular keyword search [6]. This application operates by executing three main tasks: search, query dissemination, and content delivery. In essence, a company in a certain area can generate Ads (associated with keywords and metatags), then put them on air via the Ad-Torrent system. Potential customers in the nearby will receive these Ads if their profiles match those of the Ads. Clearly, potential customers have to subscribe the Ad-Torrent application providing keywords and tags that identify their profiles (i.e., interests, description of their electronic equipments, working experience, etc.).

Selfish application 2. New devices are more and more frequently endowed with communication capabilities. These communication capabilities could be exploited, for instance, to protect one's patrimony. As an example, in case of alarm a security camera could be remotely controlled from a smart phone to carefully check a certain area. Moreover, a lost or stolen digital camera (or a camera endowed phone) could be remotely controlled to transmit back its position, stored personal pictures, or even new pictures just taken from its current location.

In their attempt to reach new customers for companies and to protect one's patrimony, these two applications can be considered as "selfish". Yet, they can be enhanced to serve also an altruistic purpose and, to demonstrate this point, we revisit examples 1 and 2.

Example 1 revisited. The general (altruism-fostering) platform attracts users because of the services that provides or just because people know that by joining it they might help somebody else; we hence assume that the sick guy, the other people at the park, and the doctor in the nearby have all subscribed the altruistic service. The sick guy has done so because he is aware that it may help him in finding quicker aid in case of necessity. People at the park have done so because they can receive Ads for products or shows that they might be interested in, or just because they run a company and would like to spread some Ads around through the aforementioned selfish application 1. The latter was also 
the aim of the doctor: he joined the service to advertise his medical specialization through is smartphone and find new customers in a very cheap way. The doctor was hence motivated in setting up his smart phone and providing all the details required to join the platform so as to be able to diffuse his Ads around. At the same time, his smart phone is now identified by the doctor's profile and can be retrieved by the platform to implement altruistic services. When the doctor's phone is reached by the aid-request message from the sick guy, it rings and loads a googlemap showing the doctor's position, the sick guy's position, and information about the problem.

Example 2 revisited. In the depicted scenario, first responders need to find a camera in the area of the accident. To this aim, they forward a message toward the emergency area. This message could be propagated in broadcast hop-by-hop until reaching the emergency area [7]. This is possible thanks to the presence of people between the first responders and the emergency area that subscribed the integrated platform: when they subscribed the service, they also accepted to provide a little percentage of their electronics' capabilities, such as some of their wireless bandwidth, in case it were necessary for some altruistic services. Once in the emergency area, the message will be received also by a security camera previously registered by its owner on the altruistic platform so as to be able to use it for his interest as described by the aforementioned selfish application 2. However, this camera can now be remotely used also by the first responders.

\section{A PLATFORM FOR ALTRUistic APPLICATIONS}

The envisioned platform can be seen as an aggregator of resources and contents that can be combined through public interfaces, APIs (Application Programmable Interfaces), web feeds, and web services. This platform will foster the creation of opportunistic applications but, at the same time, their technological means and information patrimonies will be also available, in an anonymous and combined way, for a use devoted to social utility.

The general functioning of the envisioned altruism-fostering platform can be explained as depicted in Figure 2. Users subscribe the services provided by the platform for different reasons: to use some fancy (selfish) applications, to altruistically participate in community development, and to benefits from the provided altruistic functionalities. Through subscription, the system profiles users and devices, as done for the doctor in example 1. These profiles are provided for each of the resources that are made available and passed to a resource classifier that categorizes them through tags to facilitate their 


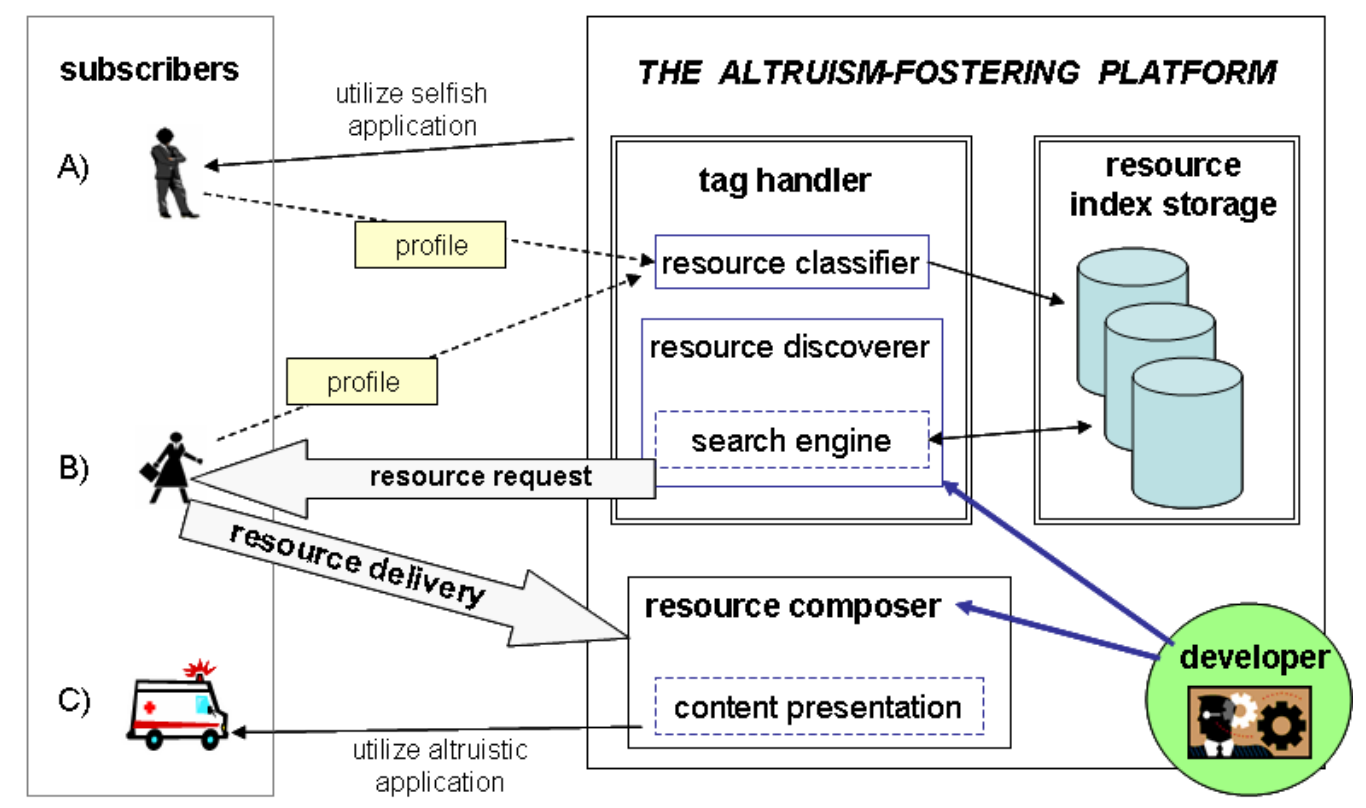

Figure 2: Logical architecture of our integrated platform.

storage. Through the use of specific APIs made available by the programming environment of the altruism-fostering platform, developers can request participants' resources and then employing them as virtual bricks to create new altruistic applications. For instance, it would be possible to develop an application that uses the communication capabilities of users spread around a certain area in order to propagate messages hop by hop till destination and, once there, activate a camera (tagged by the system at subscription time) to transmit back a video stream, as done in example 2. This can be realized by the software developer via a simple command that implies the use of a camera which is featured with certain properties (location, etc); the resource composer module highlighted in Figure 2 will take care of retrieving that resource and other necessary networking resources in order to connect the camera with the application and exchange commands and video flows. Furthermore, the system will also take care of transcoding the video flow (or, in general, any data generated by the application/resource in use) into the most appropriate format for the final user's device.

The creation of this system is significantly more complex than the development of a specific system for a single use over a given mass media; main issues and corresponding solution guidelines are: 


\section{Freedom from the technological device}

To avoid social and development inequalities, the infrastructure must integrate in a transparent way the most popular communication platforms (Internet, mobile, television); moreover, contents must be adapted depending on the distribution context, the current state of involved resources, and by considering users' preferences, interaction capabilities, localization, movements, hardware/software characteristics of the access device, connectivity typology.

\section{Service personalization and media integration}

Current applications are generally designed considering users without any physical difficulty and that have a deep technological background; as well as it is supposed that they can utilize devices with sophisticated multimedia/networking features and can easily interact through very small devices. This is clearly not a comprehensive perspective of all members of our society. Applications should interact with users through interfaces designed considering accessibility and usability [8].

\section{Localization systems}

The localization of a user or a device is a necessary condition for many altruistic applications. To this aim, approaches known to the scientific community could be exploited (GPS, RFID, localization through radio waves) in order to localize in real-time mobile users, devices, and resources.

\section{Resource donation system}

The envisioned platform clearly needs to be supported by users that donated part of their resources. A motivation for these donations could be the awareness of contributing to create a better world but could also be embodied by more materialistic rewards; for instance, the possibility to use the aforementioned selfish applications 1 and 2. Once convinced, a user can just subscribe the system which will also involve providing information to create a profile and installing a software on his device. This software will enable both the motivating selfish applications offered for free to subscribers and the resource sharing mechanism.

\section{Resource/Information classification and research}

The infrastructure has to allow users to provide and retrieve resources. Therefore, resource classification becomes fundamental. Given the great variety of resources (e.g., medical data, traffic 
information, language translator, wireless connectivity, cellphone camera), the use of classic formal classification could not be adequate; rather, collaborative tagging mechanisms (folksonomies) could be involved, allowing users to tag both information and resources without any formal constraints.

\section{Development of altruistic applications}

Through the discussed integrated platform, novel applications able to act on the structure of the society should be easily deployable. Generally speaking, applications like road safety, social networks, health and public emergency, management of climatic events, and remote education can be beneficial to the society. To this aim, the reasons moving developers toward creating these altruistic applications are many; indeed, the viability of alternative forms of software development, based on voluntary collaboration, has already been proven in practice by the success of many open source projects and other initiatives such as Wikipedia $[9,10]$. Indeed, creating new altruistic applications through the proposed integrated platform may represent the next great frontier of peer production communities.

To put into practice the aforementioned logical functionalities, it is necessary to use, develop, adapt, and integrate several existing technologies for content management and distribution, such as those reported in Figure 3. By considering a top down approach, technologies for the treatment and the codification of the information become fundamental to guarantee the production, presentation, modification, and enrichment of contents. In details, it is important to manage the meta-information describing contents so as to facilitate their recovery and utilization. Finally, the distribution of both meta-data and contents has to be handled by effective communication mechanisms, so as to be diffused through any network typology.

\section{CONCLUSION}

Through the envisioned integrated platform, able to recycle Web 2.0's waste into altruistic bricks, it will be possible to factually create an altruism-fostering platform able to transform the Internet technology into a fully democratizing tool. Consequently, the cultural and social patrimony will be increased, contributing to a sustainable development of the world. If Web 2.0 is based on the saying "yield knowledge and you will acquire power", the motto at the base of altruistic networks goes beyond: "yield resources and you will foster the entire society, including yourself". 


\begin{tabular}{|c|c|c|c|c|c|c|}
\hline \multicolumn{5}{|c|}{ CONTENT PRESENTATION } & \multicolumn{2}{|c|}{ XML-based protocols } \\
\hline MHP & InkXML & EMMA & SRGS & SSML & MPEG & SMIL \\
\hline \multicolumn{2}{|c|}{ Speech-to-Text } & ext-to-Speech & & Services & AJAX & XSLT \\
\hline \multicolumn{2}{|c|}{ RESOURCE TAGGING } & Social Nety & work $\mathrm{Ar}$ & lysis & & \\
\hline ACCMD & ACCLIP & UAProf & & & \multicolumn{2}{|c|}{ Folksonomies } \\
\hline \multicolumn{2}{|c|}{ XML } & & $\mathrm{RDF}$ & & \multicolumn{2}{|c|}{ Profiling Systems } \\
\hline \multirow{2}{*}{\multicolumn{2}{|c|}{$\begin{array}{l}\text { TAG AND INFO } \\
\text { DISTRIBUTION }\end{array}$}} & ynchronization & & sting & gossiping & $\mathrm{P} 2 \mathrm{P}$ \\
\hline & & & & NET & ANET & \\
\hline \multicolumn{2}{|c|}{$\begin{array}{ll}\mathrm{DVB}-\mathrm{T} / \mathrm{C} / \mathrm{S} / \mathrm{H} & \mathrm{DMB} \\
\end{array}$} & GPRS & UMTS & 802.1 & \multicolumn{2}{|c|}{ wired networks } \\
\hline
\end{tabular}

Figure 3: Underlying technologies supporting altruistic applications in the integrated broadband/broadcast platform.

\section{References}

[1] Roccetti, M., Ferretti, S., Palazzi, C. E., Furini, M., and Salomoni, P., Riding the Web evolution: from egoism to altruism. In Proceedings of the IEEE Consumer Communication 6 Networking 2008 (January 2008).

[2] Gill, K. S., Knowledge and the Post-industrial Society. in K. S. Gill (ed.), Information Society, Springer, New York, 1996.

[3] Bauman, Z., The individualized society. Cambridge: Polity (publisher), 2001.

[4] Castells, M., The Information Age: Economy, society and culture; Vol.I: The Rise of the Network Society. Cambridge MA. Oxford UK: Blackwell Publishers, 1996.

[5] Cialdini, R. B., Altruism or egoism? That is (still) the question. Psychological Inquiry 10, 2 (April 1991).

[6] Nandan, A., Das, S., Zhou, B., Pau, G., and Gerla, M., Ad-Torrent: Digital billboards for vehicular networks. In Proceedings of the IEEE/ACM International Workshop on Vehicle-to-Vehicle Communications (V2VCOM) (July 2005). 
[7] Roccetti, M., Gerla, M., Palazzi, C. E., Ferretti, S., and Pau, G., First Responders' Crystal Ball: How to Scry the Emergency from a Remote Vehicle. In Proceedings of the IEEE NetCri 07 IPCCC 2007 (April 2007).

[8] Kon, F., Costa, F., Blair, G., and Campbell, R. H., The case for reflective middleware. Communications of the ACM 45, 6 (June 2002).

[9] Hars A., Ou S., Working for Free? - Motivations of Participating in Open Source Projects. In Proceedings of the 34th Hawaii International Conference on System Sciences (January 2001).

[10] Nov O., What Motivates Wikipedians? Communications of the ACM 50, 11 (November 2007). 